\title{
Perinatal consequences of disproportionate fetal trunk growth
}

\author{
Ralph L. Cavalieri ${ }^{1 *}$, Suzanne Laroche ${ }^{2}$, Wayne R. Cohen ${ }^{2}$ \\ ${ }^{1}$ Departments of Obstetrics and Gynecology, Jamaica Hospital Medical Center, Weill Cornell Medical College, New York, USA \\ ${ }^{2}$ Department of Obstetrics \& Gynecology and Women's Health, Albert Einstein College of Medicine, New York, USA \\ Email: ${ }^{\text {rlc33663@gmail.com }}$
}

Received 14 April 2012; revised 13 May 2012; accepted 22 May 2012

\begin{abstract}
Objective: To identify the impact of an abnormally large neonatal chest circumference relative to head circumference on labor and neonatal morbidity. Methods: We used a retrospective cohort design to study 54 obstetric cases in which the neonatal thoracic circumference was $\geq 2.5 \mathrm{~cm}$ greater than that of the head. For each case we sought controls with a smaller thorax-head circumference difference. Ninety-seven controls were matched with their respective cases for birth weight, parity, maternal body mass index (BMI), and maternal ethnicity. Results: Cases had significantly smaller heads and larger trunks than controls $(\mathrm{P}<0.0001)$. Cases were twice as likely $(39 \%$ vs $19 \%$, $P=0.007)$ to require admission to the neonatal intensive care unit. There was no significant difference between cases and controls in the frequency of shoulder dystocia, long second stage, or long deceleration phase of labor. However, compound presentations occurred more frequently in the cases than in controls $(5.5 \%$ vs $0 \%, P=0.044)$. Conclusion: Babies with disproportionately large trunk growth were at risk for requiring neonatal intensive care and for compound presentation.
\end{abstract}

Keywords: Neonatal Morbidity; Fetal Dysmorphism; Somatic Asymmetry; Labor Abnormality; Deceleration Phase; Shoulder Dystocia; Compound Presentation; Trunk Circumference; Head Circumference

\section{INTRODUCTION}

The fetal head is normally about $2 \mathrm{~cm}$ larger in circumference than the chest [1-3], a proportionality that fosters unimpeded vaginal delivery of cephalic-presenting fetuses. A discrete aberrant fetal growth pattern has been described in which the trunk is disproportionately large relative to the head, and some studies have implicated a

"Corresponding author. large thorax/head size ratio as a risk factor for shoulder dystocia, Erb palsy, and various neonatal morbidities [4-9]. Modanlou et al. even recommended attempting to identify those fetuses with disproportionately large trunks by ultrasound and offering cesarean delivery to their mothers [10].

A large fetal chest might encumber descent of the fetus and hinder delivery, thereby predisposing to dysfunctional labor, shoulder dystocia, and consequent neonatal morbidity. It is, however, not clear whether the adversity that some investigators have found to be associated with a disproportionately large fetal chest is a consequence of mechanical interference with parturition caused by that growth discrepancy per se, or results from the same metabolic conditions that caused the asymmetry. If the latter were true, disproportionate chest growth might have consequences more enduring than those related to difficult passage through the birth canal.

To help clarify the role of disproportionate thoracic circumference (TC) growth on labor and the neonate, we compared a group of neonates in whom the TC was $\geq 2.5$ $\mathrm{cm}$ larger than the head circumference $(\mathrm{HC})$ to controls with a smaller TC-HC difference. We hypothesized that a large TC-HC difference would be associated with the need for neonatal intensive care unit (NICU) admission and with labor disorders that are risk factors for shoulder dystocia.

\section{MATERIALS AND METHODS}

The study was granted expedited review approval by the Institutional Review Board of Jamaica Hospital Medical Center. We used a retrospective cohort design to compare a group of cases with a large TC-HC difference with matched controls. The primary outcome variables were NICU admission and the presence of a prolonged deceleration phase of labor. The latter is the labor dysfunction most strongly associated with the subsequent occurrence of shoulder dystocia $[4,11,12]$. Sample size calculations revealed that to have an $80 \%$ power to detect a tripling in 
the frequency of either outcome (from an estimated $10 \%$ to $30 \%$ ) with an alpha of 0.05 would require a sample of 82 subjects.

A $\log$ of the characteristics, including head and chest circumference measurements determined by the admitting nurse, of all infants admitted to the well-baby nursery and the NICU was kept routinely at the study hospital. To identify cases we surveyed these logs for the period January 1, 2004 through December 31, 2008, during which there were 14,843 total deliveries.

One hundred and five term ( $\geq 37 \mathrm{wks}$ ) singleton infants $(0.7 \%)$ without major anomalies were identified with a chest circumference at least $2.5 \mathrm{~cm}$ larger than that of the head. Controls were chosen for each case by seeking from the computerized obstetric birth log the two deliveries in closest proximity to the case that matched it for birth weight $( \pm 5 \%)$, parity (nullipara or multipara), BMI $\left(<29\right.$ or $\left.\geq 29 \mathrm{~kg} / \mathrm{m}^{2}\right)$, and maternal ethnicity (black, white, Hispanic, south Asian, Asian, Indian/Guyanese, Guyanese, other). Forty-three cases could be matched on all four criteria with two controls; an additional 11 cases could be matched with only one control. The study sample therefore consisted of 54 cases and 97 matched controls. The matching variables are known to influence labor and neonatal outcome $[4,13]$. The matching was thus carried out to help isolate the effects of a large TC-HC difference on labor and neonatal condition.

The medical records of all cases and controls were reviewed. It was determined whether the baby was admitted to the well-baby nursery or the NICU. Indications for NICU admission and length of stay were documented, as were Apgar scores, gestational age, birth weight, and sex. Data abstracted from maternal records included age, BMI, pregnancy and labor complications, delivery mode, presence of shoulder dystocia, birth injury, and labor dysfunction. Labor curves were constructed from the data in the record by one author blinded to whether the subject was a case or a control. Dysfunctional labor patterns were categorized according to the method of Fried$\operatorname{man}[13]$.

All data were entered into a statistical software program for analysis (JMP 5.0; SAS Institute, Inc., Cary, North Carolina). Differences between cases and controls were sought using Student's t-test for continuous variables and chi square analysis for categorical data. Fisher's exact test was used when cell numbers were less than five. A probability level of 0.05 was considered to be significant. Data are presented as means \pm standard deviation, except for the quantitative labor data (durations of deceleration phase and second stage). Because these were not normally distributed, they were expressed as medians, with $10^{\text {th }}$ and $90^{\text {th }}$ percentile limits. Differences between cases and controls for these variables were assessed with the nonparametric Mann-Whitney rank sum test.

\section{RESULTS}

Cases and controls were not different with respect to the matched variables, or to the distribution of diabetes (gestational or pregestational), hypertension, gestational age, and neonatal sex (Table 1). The proportion of diabetics was not different in the case and control groups,

Table 1. Characteristics of cases and controls.

\begin{tabular}{cccc}
\hline Variable & Cases $(\mathrm{n}=54)$ & Controls (n=97) & P value \\
\hline${ }^{*}$ BMI $\left(\mathrm{kg} / \mathrm{m}^{2}\right)$ & $28.3 \pm 5.4$ & $27.4 \pm 6.2$ & 0.385 \\
${ }^{*}$ Nulliparity n (\%) & $22(41 \%)$ & $39(40 \%)$ & 0.542 \\
Maternal age (yr) & $28.3 \pm 5.4$ & $27.4 \pm 6.2$ & 0.385 \\
Diabetes mellitus (\%) & $4 / 54(7.4 \%)$ & $7 / 97(7.2 \%)$ & 0.993 \\
Birth weight $<4000 \mathrm{~g}$ & $3 / 37(8.1 \%)$ & $6 / 70(8.6 \%)$ & 0.999 \\
Birth weight $\geq 4000 \mathrm{~g}$ & $1 / 17(5.9 \%)$ & $1 / 27(3.7 \%)$ & 0.859 \\
Hypertension (\%) & $4 \%$ & $4 \%$ & 0.460 \\
${ }^{*}$ Birth weight (g) & $3749 \pm 496$ & $3685 \pm 505$ & 0.682 \\
Female sex n (\%) & $27(50 \%)$ & $46(47 \%)$ & $<0.0001$ \\
Thoracic circumference (cm) & $36.3 \pm 1.6$ & $34.3 \pm 1.8$ & $<0.0001$ \\
Head circumference (cm) & $33.5 \pm 1.6$ & $34.9 \pm 1.4$ & $<0.0001$ \\
TC-HC (cm) & $2.84 \pm 0.7$ & $-0.59 \pm 1.3$ & $<0.0001$ \\
TC/HC ratio & $1.09 \pm 0.02$ & $0.98 \pm 0.04$ & \\
\hline
\end{tabular}

"Matched variables; Racial/ethnic matching not shown. Each case matched with one of 8 racial/ethnic groups listed in text; TC, thoracic circumference; HC, head circumference; BMI, body mass index. 
regardless of whether or not the newborn weighed $>4000$ g. The differences in thorax and head circumference measurements between the two groups were due both to significantly smaller heads $(\mathrm{P}<0.0001)$ and to larger trunks $(\mathrm{P}<0.0001)$ in the cases compared to the controls.

Although there was no difference in the frequency of low Apgar scores, cases were significantly more likely to require NICU admission than controls (39\% vs 19\%, P = 0.007) (Table 2). All of the cases admitted to the NICU had a TC/HC ratio above the median for their group (1.078) and one-third had a ratio above the $75^{\text {th }}$ percentile. NICU admissions in the cases were primarily (62\%) for the diagnosis "rule out sepsis", although no baby was ultimately diagnosed with infection or sepsis. By contrast, only $33 \%$ of controls who went to the NICU were admitted for that diagnosis. The remaining common NICU admission diagnoses did not differ significantly between cases and controls, and were mild respiratory problems (5\% in each group) and hyperbilirubinemia (29\% cases, $44 \%$ controls).

The lengths of the deceleration phase and second stage of labor did not differ significantly between the cases and controls, although there was a tendency for both to be longer in the cases. The frequency with which any documentable first stage or second stage labor disorder occurred was similar between the groups, as was the presence of a long deceleration phase or long second stage (Table 2). The cesarean delivery rate was high in both groups ( $41 \%$ in cases and $46 \%$ in controls, $\mathrm{P}=$ $0.310)$, compared to an overall rate of $32 \%$ on the whole obstetric service during the study interval. The cesarean section rate increased directly with birth weight in both cases and controls. Shoulder dystocia occurred in four cases $(13 \%)$ and in three controls $(6 \%)$, a difference that did not reach significance $(\mathrm{P}=0.245)$. There were three compound presentations among the cases (5.6\%) and none in controls, a significant difference $(\mathrm{P}=0.044)$. All of these cases involved an arm prolapsed before the head, and all occurred in cases with a $\mathrm{TC} / \mathrm{HC}$ ratio above the $75^{\text {th }}$ percentile for the cases.

\section{DISCUSSION}

Somatic asymmetry in which the trunk becomes disproportionately large relative to the head during fetal life was initially observed as a feature of macrosomic babies of diabetic mothers [14]. It is now recognized that this pattern of disproportionate growth occurs in nondiabetics as well and, although it is most common in large birth weight neonates, it can occur also in those of normal weight [4]. In fact, in our own case series only $7 \%$ of the babies with a large $\mathrm{TC} / \mathrm{HC}$ ratio were from mothers with diabetes, and only $31 \%$ (17/54), weighed more than 4000 g (Table 1).

Thus, the operant factors in the production of asymmetric chest and head growth in these fetuses may be more complicated than can be explained by the excess

Table 2. Labor and neonatal outcomes.

\begin{tabular}{|c|c|c|c|}
\hline Variable & Cases & Controls & $P$ value \\
\hline \multicolumn{4}{|l|}{ Deceleration phase $^{* *}$} \\
\hline Nulliparas & $111(17,142)$ & $79(22,153)$ & 0.139 \\
\hline Multiparas & $55(11,106)$ & $41(13,121)$ & 0.191 \\
\hline \multicolumn{4}{|l|}{ Stage II ${ }^{* *}$} \\
\hline Nulliparas & $68(1,123)$ & $33(9,108)$ & 0.442 \\
\hline Multiparas & $11(1,97)$ & $11(2,34)$ & 0.418 \\
\hline Labor dysfunction ${ }^{*}$ & $19 / 40(48 \%)$ & $27 / 67(40 \%)$ & 0.299 \\
\hline Long deceleration phase & $12 / 33(36 \%)$ & $17 / 60(28 \%)$ & 0.284 \\
\hline Long stage $\mathrm{II}^{\ddagger}$ & $3 / 31(10 \%)$ & $3 / 51(6 \%)$ & 0.409 \\
\hline Shoulder dystocia ${ }^{* * *}$ & $4 / 32(13 \%)$ & $3 / 52(6 \%)$ & 0.245 \\
\hline Compound presentation & $3(5.5 \%)$ & $0(0 \%)$ & 0.044 \\
\hline Cesarean delivery & $22(41 \%)$ & $45(46 \%)$ & 0.502 \\
\hline NICU admission & $21(39 \%)$ & $18(19 \%)$ & 0.007 \\
\hline 5-min Apgar score $<7$ & $0(0 \%)$ & $1(1 \%)$ & 0.762 \\
\hline
\end{tabular}

* Presence of any active phase or second stage graphic labor abnormality; ${ }^{* *}$ Expressed as median $\left(10^{\text {th }}, 90^{\text {th }}\right.$ percentile $)$. Excludes subjects who had cesarean delivery before labor or during the latent phase; ${ }^{* * *}$ Vaginal deliveries only; ${ }^{\dagger}>120 \mathrm{~min}$ in nullipara, $>60$ min in multipara. 
insulin and insulin-like growth factors that are important governors of macrosomia in the fetus of a diabetic mother. The consequences of chest/head disproportion are also uncertain, but our results suggest there is reason for concern about the perinatal implications of this aberrant growth pattern.

In this study we addressed primarily the general question of whether this uneven growth relationship affected the newborn's adaptation to extrauterine life. To maximize the chance to identify an effect of chest and head growth imbalance per se, we matched our disproportionate growth cases with controls for parity, birth weight, BMI and race, all factors known to influence the likelihood of labor abnormalities and newborn morbidity. In addition, the cases and controls had equal proportions of women with hypertension and diabetes, and equivalent newborn sex ratios, factors with the potential to influence outcome and the need for NICU admission. Despite this balance in risk factors between case and control groups, the cases had twice the rate of NICU admission than did the controls, and the admitted cases tended to be those with the largest TC-HC differences. Almost all of these admissions were for a constellation of nonspecific signs that made the neonatologists sufficiently uneasy about a baby's condition to admit it to the intensive care unit for observation. These signs included lethargy, reduced tone, and mild respiratory difficulties without any obvious source.

No baby had sepsis, respiratory distress syndrome, Erb palsy or other birth injury, and all the Apgar scores of the cases were above seven at one and five minutes. Nevertheless, neonatologists recognized aberrant behavior or worrisome signs and symptoms in case babies that led to their NICU admission. These decisions were not attributable to differences in maternal medical risks (diabetes, hypertension and obesity) or to differences in birth weight, parity, race, delivery mode, or Apgar scores. Moreover, the babies' body measurements were not known until after the decision for NICU admission was made. Our results are consistent with the notion that there is something about fetuses with this growth disproportion that affects their early neonatal adaptation.

Somatic asymmetry may be an important risk factor in the occurrence of shoulder dystocia. For example, Modanlou et al. found an increased incidence of shoulder dystocia when the chest circumference was $1.6 \mathrm{~cm}$ larger than that of the head [10]. Our study shows that this disproportion occurs in many macrosomic as well as normal-weight babies, whether or not their mother had glucose intolerance. Macrosomic neonates usually have a head and chest of equal size $[15,16]$, or have a head substantially smaller than the chest [16], the opposite of what is observed in most normal birth weight babies [2, $3]$. Our sample size was inadequate to assess the differ- ence in the frequency of Erb palsy, or even shoulder dystocia between cases and controls, because the prevalence of both is low (about 20/1000 for shoulder dystocia and 2.5/1000 for brachial plexus injury). Instead, we used as proxies labor disorders strongly associated with the diagnosis of neonatal Erb palsy: prolonged deceleration phase of labor and long second stage [4]. The observed frequency of these abnormalities was not significantly different in our cases and controls. There was, however, a trend toward a longer deceleration phase and a longer second stage in cases. The failure to find a significant difference could relate to our sample size, because the frequency of long deceleration phase was considerably greater than we anticipated when the sample size calculation was done. Also, the very high cesarean rate eliminated many cases from consideration of the deceleration phase because they were delivered prior to the terminal portion of the first stage. Finally, it may take an unusually large TC-HC difference to affect fetal expulsion in this manner. Our case with the largest discrepancy $(6 \mathrm{~cm})$ had a severe shoulder dystocia. Thus, this issue deserves further study in larger samples.

The usual prevalence of compound presentation is about one in 1200 deliveries. Our finding of three compound presentations in the cases was, therefore, not anticipated. While one cannot draw firm conclusions from so few cases, our incidence of $5.5 \%$ was striking. We speculate that the relatively small head in these cases allowed space for the arm to prolapse. Further corroboration of this possibility is necessary.

We conclude from our observations that babies with a disproportionately large chest in comparison to their head constitute a discrete group at increased risk. The reasons for their apparent maladaptation to early neonatal life are unknown, as are the potential long-term consequences of this growth asymmetry. It is noteworthy that not only are the chests of these babies especially large, but their heads are significantly smaller than expected as well. Diminished head size raises the possibility of altered brain growth or function. We have identified an unusual fetal growth pattern, the perinatal and long-term consequences of which merit further investigation.

\section{REFERENCES}

[1] Catalano, P.M., Thomas, A., Huston-Presley, L. and Amini, S.B. (2003) Increased fetal adiposity: A very sensitive marker of abnormal in utero development. American Journal of Obstetrics \& Gynecology, 189, 1698-1704. doi:10.1016/S0002-9378(03)00828-7

[2] Catalano, P.M., Drago, N.M. and Saeid, B.A. (1995) Factors affecting fetal growth and body composition. American Journal of Obstetrics \& Gynecology, 172, 1459-1463. doi:10.1016/0002-9378(95)90478-6 
[3] Catalano, P.M., Thomas, A.J., Avallone, D.A. and Amini S.B. (1995) Anthropometric estimation of neonatal body composition. American Journal of Obstetrics \& Gynecology, 173, 1176-1181. doi:10.1016/0002-9378(95)91348-3

[4] Weizsaecker, K., Deaver, J.E. and Cohen, W.R. (2007) Labour characteristics and neonatal Erb's palsy. British Journal of Obstetrics and Gynaecology, 114, 1003-1009. doi:10.1111/j.1471-0528.2007.01392.x

[5] Bahar, A.M. (1996) Risk factors and fetal outcome in cases of shoulder dystocia compared with normal deliveries of similar birthweight. British Journal of Obstetrics and Gynaecology, 103, 868-872. doi:10.1111/j.1471-0528.1996.tb09904.x

[6] Miller, R.S., Devine, P.C. and Johnson, E.B. (2007) Sonographic fetal asymmetry predicts shoulder dystocia. Journal of Ultrasound in Medicine, 26, 1523-1528.

[7] Elliott, J.P., Garite, T.J., Freeman, R.K., McQuown, D.S. and Patel, J.M. (1982) Ultrasonic prediction of fetal macrosomia in diabetic patients. Obstetrics \& Gynecology, 60 , 159-162.

[8] Cohen, B.F., Penning, S., Ansley, D., Porto, M. and Garite, T. (1999) The incidence and severity of shoulder dystocia correlates with a sonographic measurement of asymmetry in patients with diabetes. American Journal of Perinatology, 16, 197-201. doi:10.1055/s-2007-993858

[9] Cohen, B., Penning, S., Major, C., Ansley, D., Porto, M. and Garite, T. (1996) Sonographic prediction of shoulder dystocia in infants of diabetic mothers. Obstetrics \& Gynecology, 88, 10-13. doi:10.1016/0029-7844(96)00067-1
[10] Modanlou, H.D., Komatsu, G., Dorchester, W., Freeman, R.K. and Bosu, S.K. (1982) Large-for-gestational-age neonates: Anthropometric reasons for shoulder dystocia. $\mathrm{Ob}$ stetrics \& Gynecology, 60, 417-423.

[11] Hopwood, H.G. (1982) Shoulder dystocia: Fifteen years' experience in a community hospital. American Journal of Obstetrics \& Gynecology, 144, 162-166.

[12] Gross, T.L., Sokol, R.J., Williams, T. and Thompson, K. (1987) Shoulder dystocia: A fetal-physician risk. American Journal of Obstetrics \& Gynecology, 156, 1408-1418.

[13] Friedman, E.A. (1978) Labor: Clinical evaluation and management. 2nd Edition, Appleton Century Crofts, New York.

[14] McFarland, M.B., Trylovich, C.G. and Langer, O. (1998) Anthropometric differences in macrosomic infants of diabetic and nondiabetic mothers. Journal of Maternal-Fetal Medicine, 7, 292-295. doi:10.1002/(SICI)1520-6661(199811/12)7:6<292::AIDMFM7>3.0.CO;2-A

[15] Koo, W.W.K., Walters, J.C. and Hockman, E.M. (2004) Body composition in neonates: Relationship between measured and derived anthropometry with dual-energy x-ray absorptiometry measurements. Pediatric Research, 56, 694-700. doi:10.1203/01.PDR.0000142587.59238.BD

[16] Salim, R., Hasanein, J., Nachum, Z. and Shalev, E. (2004) Anthropometric parameters in infants of gestational diabetic women with strict glycemic control. American Journal of Obstetrics \& Gynecology, 104, 1021-1024. doi:10.1097/01.AOG.0000143821.00194.ad 